\title{
A BMO TYPE CHARACTERIZATION OF WEIGHTED LIPSCHITZ FUNCTIONS IN TERMS OF THE BEREZIN TRANSFORM
}

\author{
Hong Rae Cho and Yeoung-Tae Seo
}

\begin{abstract}
The Berezin transform is the analogue of the Poisson transform in the Bergman spaces. Dyakonov characterize the holomorphic weighted Lipschitz function in the unit disk in terms of the Poisson integral. In this paper, we characterize the harmonic weighted Lipschitz function in terms of the Berezin transform instead of the Poisson integral.
\end{abstract}

\section{Introduction}

A continuous function $\omega:(0, \infty) \rightarrow \mathbb{R}$ with $\lim \sup _{t \rightarrow 0^{+}} \omega(t)=0$ will be called a modulus of continuity, if $\omega(t)$ is non-negative and nondecreasing. If, in addition, there is a constant $C(\omega)>0$ such that

$$
\int_{0}^{t} \frac{\omega(s)}{s} d s+t \int_{t}^{\infty} \frac{\omega(s)}{s^{2}} d s \leq C(\omega) \omega(t)
$$

where $0<t<1$, then we say that it is regular. Given a modulus of continuity $\omega$ and $E \subset \mathbb{R}^{N}$, we define the weighted Lipschitz space by

$$
\Lambda_{\omega}(E)=\{f:|f(x)| \leq M,|f(x)-f(y)| \leq M \omega(|x-y|), x, y \in E\}
$$

with norm the smallest $M$. Weighted Lipschitz spaces have been studied by many authors (see $[5,6,8,9,10,12,13]$, and references in their papers).

Let $\mathbf{D}$ be the unit disk $\{z \in \mathbb{C}:|z|<1\}$ and $\mathbf{T}=\{z \in \mathbb{C}:|z|=1\}$ its boundary in [8], Dyakonov proved the following.

Received April 26, 2005.

2000 Mathematics Subject Classification: 31A05, 31A10.

Key words and phrases: weighted Lipschitz space of harmonic functions, Berezin transform, a regular modulus of continuity.

This work was supported by Korea Research Foundation Grant (KRF-2004-002C00013). 
THEOREM 1. Let $f$ be a holomorphic function on $\mathbf{D}$ that is continuous up to $\mathbf{T}$. If both $\omega$ and $\omega^{2}$ are regular, then

$$
\|f\|_{\Lambda_{\omega}(\mathbf{T})} \sim \sup _{\mathbf{D}} \frac{1}{\omega(1-|z|)}\left[\mathcal{P}\left(|f|^{2}\right)(z)-|f(z)|^{2}\right]^{1 / 2} .
$$

Here $\mathcal{P}$ is the Poisson integral operator defined by

$$
\mathcal{P} g(z)=\int_{\mathbf{T}} g(\zeta) \frac{1-|z|^{2}}{|\zeta-z|^{2}} d \sigma(\zeta), \quad z \in \mathbf{D}
$$

and by

$$
\mathcal{P} g(\zeta)=g(\zeta), \quad \zeta \in \mathbf{T} .
$$

For every function $h \in L^{1}(\mathbf{D}, d A)$; we define

$$
\mathbb{B} h(z)=\int_{\mathbf{D}} \frac{\left(1-|z|^{2}\right)^{2}}{|1-z \bar{w}|^{4}} h(w) d A(w), \quad z \in \mathbf{D},
$$

where $d A$ is the normalized area measure on $\mathbf{D}$. The operator $\mathbb{B}$ will be called the Berezin transform. It is remarkable that $h \in L^{1}(\mathbf{D}, \mathbf{d A})$ is harmonic if and only if $\mathbb{B} h=h$ (see [1]).

In this paper, we prove the Berezin transform version of Theorem 1 as following.

THEOREM 2. Let $\omega$ be a regular modulus of continuity. Let $h$ be harmonic in $\mathbf{D}$. Consider the equalities

$$
\begin{aligned}
& M_{0}(h)=\sup _{z \in \mathbf{D}}\left[\frac{(1-|z|)}{\omega(1-|z|)}|\nabla h(z)|\right], \\
& M_{1}(h)=\sup _{z \in \mathbf{D}} \frac{1}{\omega(1-|z|)} \mathbb{B}(|h-h(z)|)(z), \\
& M_{2}(h)=\sup _{z \in \mathbf{D}} \frac{1}{\omega(1-|z|)}\left[\mathbb{B}\left(|h|^{2}\right)(z)-|(\mathbb{B} h)(z)|^{2}\right]^{1 / 2} .
\end{aligned}
$$

(i) Let $h \in L^{1}(\mathbf{D})$. If $h$ is harmonic in $\mathbf{D}$, then we have

$$
M_{0}(h) \sim M_{1}(h) .
$$

(ii) Let $h \in L^{2}(\mathbf{D})$. If $h$ is harmonic in $\mathbf{D}$, then we have

$$
M_{0}(h) \sim M_{2}(h) .
$$

Let $\omega$ be a regular modulus of continuity. By the same argument as the proof of Hardy-Littlewood lemma for harmonic Lipschitz functions, . we can prove that

$$
\|h\|_{\Lambda_{\omega}(\mathbf{D})} \sim \sup _{\mathbf{D}}|h|+M_{0}(f)
$$


for functions $h$ harmonic in D. By (1) and Theorem 2, we have the following.

COROllary 3. Let $\omega$ be a regular modulus of continuity. Let $h$ be harmonic in $\mathbf{D}$.

(i) If $h \in L^{1}(\mathbf{D})$, then we have

$$
\|h\|_{\Lambda_{\omega}(\mathbf{D})} \sim \sup _{z \in \mathbf{D}}|h|+M_{1}(h) .
$$

(ii) If $h \in L^{2}(\mathbf{D})$, then we have

$$
\|h\|_{\Lambda_{\omega}(\mathbf{D})} \sim \sup _{z \in \mathbf{D}}|h|+M_{2}(h) .
$$

The Berezin transform was introduced by Berezin in [3] and [4] and most applications of the Berezin transform so far have been in study of Hankel and Toeplitz operators (see [16]). The characterization of the $\mathrm{BMO}$ function in the Bergman metric by the Berezin transform was begun by Zhu in his thesis [15] and then developed by Békollé, Berger, Coburn, and Zhu in [2]. We state the results in Section 2. Their results motivated the authors to consider the characterization of the harmonic Lipschitz function in terms of the Berezin transform.

\section{Preliminaries}

LEMMA 4. Let $\Omega$ be an open subset in $\mathbb{R}^{N}$. Let $\ell$ be a line segment lying entirely in $\Omega$. Suppose that $f: \Omega \rightarrow \mathbb{R}$ is continuous and

$$
\liminf _{x \rightarrow x_{0}} \frac{\left|f(x)-f\left(x_{0}\right)\right|}{\left|x-x_{0}\right|}=0, \quad \text { for each } \quad x_{0} \in \ell .
$$

Then $f$ is constant on $\ell$.

Proof. Let $a, b \in \Omega$ and $\ell=\{t a+(1-t) b: 0 \leq t \leq 1\}$. Suppose that $f$ is not constant on $\ell$. Then there are $t_{1}, t_{2} \in[0,1]$ such that

$$
f\left(a_{0}\right)=f\left(t_{1} a+\left(1-t_{1}\right) b\right)<f\left(t_{2} a+\left(1-t_{2}\right) b\right)=f\left(b_{0}\right) .
$$

We may assume that $t_{1}<t_{2}$. We choose a line $L$ with slope $\alpha>0$ separating these two points $\left(a_{0}, f\left(a_{0}\right)\right),\left(b_{0}, f\left(b_{0}\right)\right)$ in the section $\{(x, s)$ : $x \in \ell, s \in \mathbb{R}\}$. Let

$$
t_{0}=\sup \{t:(t a+(1-t) b, f(t a+(1-t) b)) \in L\} .
$$


Then for $t_{0}<t<1$ the point $(t a+(1-t) b, f(t a+(1-t) b)$ lies above $L$. Thus we have

$$
\liminf _{t \rightarrow t_{0}^{+}} \frac{\left.\mid f(t a+(1-t) b))-f\left(t_{0} a+\left(1-t_{0}\right) b\right)\right) \mid}{\left|\left(t-t_{0}\right) a+\left(t-t_{0}\right) b\right|} \geq \alpha>0 .
$$

This is a contradiction.

Corollary 5. Let $\Omega$ be a connected open subset in $\mathbb{R}^{N}$. If

$$
\liminf _{x \rightarrow x_{0}} \frac{\left|f(x)-f\left(x_{0}\right)\right|}{\left|x-x_{0}\right|}=0, \quad \text { for each } x_{0} \in \Omega,
$$

then $f$ is constant on $\Omega$.

Proof. Let $a, b \in \Omega$. Since $\Omega$ is connected, $a$ and $b$ can be joined by a polygonal curve lying entirely in $\Omega$. By Lemma $4, f(a)=f(b)$.

Proposition 6. Let $\omega$ be a modulus of continuity. Let $\Omega$ be a connected open subset in $\mathbb{R}^{N}$. If $\liminf \operatorname{int}_{t \rightarrow 0^{+}}(\omega(t) / t)=0$, then $\Lambda_{\omega}(\Omega)=$ \{constants\}.

Proof. We have

$$
\liminf _{y \rightarrow 0} \frac{|f(x+y)-f(x)|}{|y|} \lesssim \liminf _{y \rightarrow 0} \frac{\omega(|y|)}{|y|}=0, \quad \text { for each } \quad x \in \Omega .
$$

By Corollary 5 , we get the result.

LEMma 7. Let $\omega$ be a regular modulus of continuity. There is $C>0$ such that

$$
\frac{\omega(\tau)}{\tau} \leq C \frac{\omega(t)}{t} \quad \text { for } \quad t \leq \tau
$$

Proof. Since $\omega$ is non-decreasing, for $t \leq \tau$, we have

$$
\frac{\omega(\tau)}{\tau}=2^{2} \tau \frac{\omega(\tau)}{(2 \tau)^{2}} \leq 2^{2} \int_{\tau}^{2 \tau} \frac{\omega(s)}{s^{2}} d s \leq 2^{2} \int_{t}^{\infty} \frac{\omega(s)}{s^{2}} d s \lesssim \frac{\omega(t)}{t} .
$$

REMARK 1. By Lemma 7, if $\omega$ is regular, then $\omega(t) / t$ is bounded below and so it excludes $\omega(t)=t^{\alpha}$ for $\alpha>1$.

COROLlary 8. Let $\omega$ be a regular modulus of continuity. Let $M \geq 1$. Let $C$ be the constant in Lemma 7. Then we get

$$
\omega(M t) \leq C M \omega(t) \text { for all } t>0 .
$$




\section{BMO in the Bergman metric}

The Bergman metric on $\mathbf{D}$, also called the hyperbolic metric or the Poincaré metric, is given by

$$
\beta(z, w)=\frac{1}{2} \log \frac{1+\left|\varphi_{z}(w)\right|}{1-\left|\varphi_{z}(w)\right|}, \quad z, w \in \mathbf{D} .
$$

For any $z \in \mathbf{D}$ and $r>0$, let

$$
D(z, r)=\{w \in \mathbf{D}: \beta(z, w)<r\}
$$

be the Bergman metric disk with center $z$ and radius $r$.

Given a function $h \in L^{1}(\mathbf{D})$, we define an averaging function $\hat{h}_{r}(z)$ on $\mathbf{D}$ as follows:

$$
\hat{h}_{r}(z)=\frac{1}{|D(z, r)|} \int_{D(z, r)} h(w) d A(w), \quad z \in \mathbf{D} .
$$

For $h \in L^{2}(\mathbf{D})$, we define the mean oscillation of $h$ at $z$ in the Bergman metric to be

$$
M O_{r}(h)(z)=\left(\frac{1}{|D(z, r)|} \int_{D(z, r)}\left|h(w)-\hat{h}_{r}(z)\right|^{2} d A(w)\right)^{1 / 2} .
$$

Let $B M O_{r}=B M O_{r}(\mathbf{D})$ denote the space of all integrable functions $h$ such that

$$
\|h\|_{r}=\sup \left\{M O_{r}(h)(z): z \in \mathbf{D}\right\}<+\infty .
$$

In fact, $B M O_{r}$ is independent of $r$ and can be described in terms of the Berezin transform.

Theorem 9 ([2], [11], [16]). Suppose $0<r<+\infty$ and that the function $h$ is locally square-integrable in $\mathbf{D}$. Then $h \in B M O_{r}$ if and only if $h \in L^{2}(\mathbf{D})$,

$$
\sup _{z \in \mathbf{D}}\left[\mathbb{B}\left(|h|^{2}\right)(z)-|\mathbb{B} h(z)|^{2}\right]^{1 / 2}<+\infty .
$$

\section{Proof of Theorem 2}

Lemma 10 ([7]). Let $1 \leq p<\infty$. Then

$$
\int_{\mathbf{D}}|h|^{p} d A \lesssim \int_{\mathbf{D}}(1-|z|)^{p}|\nabla h(z)|^{p} d A+|h(0)|^{p}
$$

for functions $h$ harmonic on $\mathbf{D}$. 
Lemma 11. Let $1 \leq p<\infty$. Let $h \in L^{p}(\mathbf{D})$ be harmonic in $\mathbf{D}$. Then

$$
|\nabla h(0)|^{p} \lesssim \int_{\mathbf{D}}|h(\zeta)-h(0)|^{p} d A(\zeta)
$$

Proof. Let $\chi: \mathbb{C} \rightarrow \mathbb{R}$ be a cut-off function satisfying:

$$
\chi \in C_{0}^{\infty}, \quad \chi \geq 0 \text { on } \mathbf{D}, \quad \chi(z)=\chi(|z|), \quad \operatorname{supp} \chi \subset \mathbf{D}, \quad \int_{\mathbf{D}} \chi d A=1 .
$$

For $\epsilon>0$, define

$$
\chi_{\epsilon}(z)=\frac{1}{\epsilon^{2}} \chi\left(\frac{z}{\epsilon}\right) \quad \text { and } \quad \mathbf{D}_{\epsilon}(z)=\{\zeta:|z-\zeta|<\epsilon\} .
$$

Since $h$ is harmonic, we have

$$
h(z)-h(0)=\int_{\zeta \in \mathbf{D}_{\epsilon}(z)}(h(\zeta)-h(0)) \chi_{\epsilon}(z-\zeta) d A(\zeta) .
$$

It follows that

$$
\begin{aligned}
|\nabla h(z)| & \leq \sup _{\zeta \in \mathbf{D}_{\epsilon}(z)}\left|\nabla \chi_{\epsilon}(z-\zeta)\right| \int_{\zeta \in \mathbf{D}_{\epsilon}(z)}|h(\zeta)-h(0)| d A(\zeta) \\
& \lesssim \frac{1}{\epsilon^{3}} \int_{\mathbf{D}}|h(\zeta)-h(0)| d A(\zeta) .
\end{aligned}
$$

Thus we have

$$
|\nabla h(0)|^{p} \lesssim \frac{1}{\epsilon^{3 p}} \int_{\zeta \in \mathbf{D}}|h(\zeta)-h(0)|^{p} d A(\zeta) .
$$

LEMMA 12. Let $\omega$ be a regular modulus of continuity. Then we have

$$
\int_{\mathrm{D}} \frac{\omega(1-|w|)}{|1-\bar{z} w|^{3}} d A(w) \lesssim \frac{\omega(1-|z|)}{1-|z|} .
$$

Proof. By the polar coordinate and the inequality in [14, Proposition 1.4.10], we have

$$
\begin{aligned}
\int_{\mathbf{D}} \frac{\omega(1-|w|)}{|1-\bar{z} w|^{3}} d A(w) & =\int_{0}^{1} \int_{\mathbf{T}} \frac{\omega(1-r)}{|1-r \bar{z} \zeta|^{3}} d \sigma(\zeta) r d r \\
& \lesssim \int_{0}^{1} \frac{\omega(1-r)}{(1-r|z|)^{2}} d r
\end{aligned}
$$

Note that $1-r|z|=(1-r)+(1-|z|)-(1-|z|)(1-r)$. Thus we have

$$
\int_{0}^{1} \frac{\omega(1-r)}{(1-r|z|)^{2}} d r=\int_{0}^{1} \frac{\omega(s)}{[s+(1-|z|)-(1-|z|) s]^{2}} d s
$$


by putting $1-r=s$. We decompose the integral by two parts as following

$$
\begin{aligned}
& \int_{0}^{1} \frac{\omega(s)}{[s+(1-|z|)-(1-|z|) s]^{2}} d s \\
= & \int_{0}^{1-|z|}+\int_{1-|z|}^{1} \frac{\omega(s)}{[s+(1-|z|)-(1-|z|) s]^{2}} d s .
\end{aligned}
$$

For the first part we have

$$
\begin{aligned}
\int_{0}^{1-|z|} \frac{\omega(s)}{[s+(1-|z|)-(1-|z|) s]^{2}} d s & \lesssim \int_{0}^{1-|z|} \frac{\omega(s)}{s[s|z|+(1-|z|)]} d s \\
& \lesssim \frac{1}{1-|z|} \int_{0}^{1-|z|} \frac{\omega(s)}{s} d s \\
& \lesssim \frac{\omega(1-|z|)}{1-|z|}
\end{aligned}
$$

Now for the second part we have

$$
\int_{1-|z|}^{1} \frac{\omega(s)}{[s+(1-|z|)-(1-|z|) s]^{2}} d s \lesssim \int_{1-|z|}^{1} \frac{\omega(s)}{s^{2}} d s \lesssim \frac{\omega(1-|z|)}{1-|z|}
$$

We use the regular condition of $\omega$ at the last step of estimates for two parts of the integral. Thus we complete the proof.

For any $z \in \mathbf{D}$, let $\varphi_{z}$ be the Möbius transformation on $\mathbf{D}$ defined by

$$
\varphi_{z}(w)=\frac{z-w}{1-\bar{z} w}, \quad z, w \in \mathbf{D} .
$$

Then the Möbius map $\varphi_{z}$ has the following properties:

$$
\begin{aligned}
\varphi_{z}^{\prime}(w) & =\frac{-\left(1-|z|^{2}\right)}{(1-\bar{z} w)^{2}}=-k_{z}(w) \\
\left(1-|w|^{2}\right) k_{z}(w) & =1-\left|\varphi_{z}(w)\right|^{2}=\frac{\left(1-|w|^{2}\right)\left(1-|z|^{2}\right)}{|1-\bar{z} w|^{2}} .
\end{aligned}
$$

The real Jacobian determinant of $\varphi_{z}$ at $w$ is

$$
\operatorname{det} J_{\mathbb{R}} \varphi_{z}(w)=\left|\varphi_{z}^{\prime}(w)\right|^{2}=\left|k_{z}(w)\right|^{2}=\frac{\left(1-|z|^{2}\right)^{2}}{|1-\bar{z} w|^{4}} .
$$


Proof of Theorem 2. (i) By the change of variable, we have that

$$
\begin{aligned}
\mathbb{B}(|h-h(z)|)(z) & =\int_{\mathbf{D}}|h(w)-h(z)| \mathbb{B}(z, w) d A(w) \\
& =\int_{\mathbf{D}}\left|\left(h \circ \varphi_{z}\right)(w)-h(z)\right| d A(w) .
\end{aligned}
$$

By Lemma 10, we have

$$
\int_{\mathbf{D}}\left|h \circ \varphi_{z}(w)-h(z)\right| d A \lesssim \int_{\mathbf{D}}\left(1-|w|^{2}\right)\left|\nabla\left(h \circ \varphi_{z}\right)(w)\right| d A .
$$

We note that

$\left|\nabla\left(h \circ \varphi_{z}\right)(w) \lesssim\right| \nabla h\left(\varphi_{z}(w) \mid k_{z}(w)\right.$ and $\left(1-|w|^{2}\right) k_{z}(w)=1-\left|\varphi_{z}(w)\right|^{2}$.

Thus it follows that

$$
\int_{\mathbf{D}}\left(1-|w|^{2}\right)\left|\nabla\left(h \circ \varphi_{z}\right)(w)\right| d A \lesssim \int_{\mathbf{D}}\left(1-|\zeta|^{2}\right)|\nabla h(\zeta)|\left|k_{z}(\zeta)\right|^{2} d A .
$$

By Lemma 12, we have

$(2)$

$$
\begin{aligned}
& \int_{\mathbf{D}}\left(1-|\zeta|^{2}\right)|\nabla h(\zeta)|\left|k_{z}(\zeta)\right|^{2} d A \\
\lesssim & \sup _{\zeta \in \mathbf{D}}\left[\frac{(1-|\zeta|}{\omega(1-|\zeta|)}|\nabla h(\zeta)|\right] \int_{\mathbf{D}} \frac{\omega(1-|\zeta|)(1-|z|)^{2}}{|1-\bar{z} \zeta|^{4}} d A(\zeta) \\
\lesssim & \sup _{\zeta \in \mathbf{D}}\left[\frac{(1-|\zeta|}{\omega(1-|\zeta|)}|\nabla h(\zeta)|\right](1-|z|) \int_{\mathbf{D}} \frac{\omega(1-|\zeta|)}{|1-\bar{z} \zeta|^{3}} d A(\zeta) \\
\lesssim & \sup _{\zeta \in \mathbf{D}}\left[\frac{(1-|\zeta|}{\omega(1-|\zeta|)}|\nabla h(\zeta)|\right] \omega(1-|z|) .
\end{aligned}
$$

Then, from (2), it follows that

$$
\frac{1}{\omega(1-|z|)} \int_{\mathbf{D}}|h(\zeta)-h(z)| \mathbb{B}(z, \zeta) d A(\zeta) \lesssim \sup _{\zeta \in \mathbf{D}}\left[\frac{(1-|\zeta|}{\omega(1-|\zeta|)}|\nabla h(\zeta)|\right] .
$$

Thus we have

$$
M_{1}(h) \lesssim M_{0}(h) .
$$

For $p=1$ we replace $h$ by $h \circ \varphi_{z}$ in Lemma 11. We have

$$
\begin{aligned}
\left(1-|z|^{2}\right)|\nabla h(z)| & \lesssim \int_{\mathbf{D}}\left|\left(h \circ \varphi_{z}\right)(w)-h(z)\right| d A(w) \\
& =\int_{\mathbf{D}}|h(\zeta)-h(z)| \mathbb{B}(z, \zeta) d A(\zeta) .
\end{aligned}
$$


Thus we have

$$
\frac{(1-|z|)}{\omega(1-|z|)}|\nabla h(z)| \lesssim \frac{1}{\omega(1-|z|)} \mathbb{B}(|h-h(z)|)(z)
$$

and hence $M_{0}(f) \lesssim M_{1}(f)$.

(ii) For $h \in L^{2}(\mathbf{D})$, as in the proof of (i), we have

$$
\begin{aligned}
\mathbb{B}\left(|h|^{2}\right)(z)-|(\mathbb{B} h)(z)|^{2} & =\int_{\mathbf{D}}\left|\left(h \circ \varphi_{z}\right)(w)-h(z)\right|^{2} d A(w) \\
& \lesssim \int_{\mathbf{D}}|\nabla h(\zeta)|^{2}\left(1-|\zeta|^{2}\right)^{2} \frac{\left(1-|z|^{2}\right)^{2}}{|1-\bar{z} \zeta|^{4}} d A(w)
\end{aligned}
$$

Thus it follows that

$$
\frac{1}{\omega(1-|z|)}\left[\mathbb{B}\left(|h|^{2}\right)(z)-|(\mathbb{B} h)(z)|^{2}\right]^{1 / 2} \lesssim \sup _{\zeta \in \mathrm{D}}\left[\frac{(1-|\zeta|)}{\omega(1-|z|)}|\nabla h(\zeta)|\right]
$$

and hence $M_{2}(h) \lesssim M_{0}(h)$.

For $p=2$ we replace $h$ by $h \circ \varphi_{z}$ in Lemma 11. Then we have

$$
\begin{aligned}
(1-|z|)^{2}|\nabla h(z)|^{2} & \lesssim \int_{\mathbf{D}}|h(\zeta)-h(z)|^{2} \mathbb{B}(z, \zeta) d A(\zeta) \\
& =\mathbb{B}\left(|h|^{2}\right)(z)-|(\mathbb{B} h)(z)|^{2}
\end{aligned}
$$

and so that

$$
\frac{(1-|z|)}{\omega(1-|z|)}|\nabla h(z)| \lesssim \frac{1}{\omega(1-|z|)}\left[\mathbb{B}\left(|h|^{2}\right)(z)-|(\mathbb{B} h)(z)|^{2}\right]^{1 / 2}
$$

Thus it follows that $M_{0}(h) \lesssim M_{2}(h)$.

\section{References}

[1] P. Ahern, M. Flores and W. Rudin, An invariant volume-mean value Property, J. Funct. Anal. 111 (1993), 380-397.

[2] Békollé, Berger, Coburn, and Zhu, BMO in the Bergman metric on bounded symmetric domains, J. Func. Anal. 93 (1990), 310-350.

[3] F. A. Berezin, Covariant and contravariant symbols of operators, Math. USSRIzv. 6 (1972), 1117-1151.

[4] __ The relation between covariant and contravariant symbols of operators on classical complex symmetric spaces, Soviet Math. Dokl. 19 (1978), 786-789.

[5] S. Bloom and G. S. De Souza, Atomic decomposition of generalized Lipschitz spaces, Illinois J. Math. 33 (1989), no. 2, 181-209.

[6] Weighted Lipschitz spaces and their analytic characterizations, Constr. Approx. 10 (1994), no. 3, 339-376.

[7] B. R. Choe, H. Koo, and H. Yi, Derivatives of Harmonic Bergman and Bloch functions on the ball, J. Math. Anal. Appl. 260 (2001), 100-123. 
[8] K. M. Dyakonov, Equivalent norms on Lipschitz-type spaces of holomorphic functions, Acta. Math. 178 (1997), 143-167.

[9] —_ Holomorphic functions and quasiconformal mappings with smooth moduli, Adv. Math. 187 (2004), 146-172.

[10] __ Strong Hardy-Littlewood theorems for analytic functions and mappings of finite distortion, Math. Z. 249 (2005), no. 3, 597-611.

[11] H. Hedenmanlm, B. Korenblum, and K. Zhu, Theory of Bergman spaces, Springer-Verlag, 2000.

[12] M. Pavlović, On Dyakonov's paper "Equivalent norms on Lipschitz-type spaces of holomorphic functions", Acta. Math. 183 (1999), 141-143.

[13] _ Introduction to Function Spaces on the Disk, Posebna izdanja 20, Mathematički Institut SANU, Beograd 2004.

[14] W. Rudin, Function theory in the unit ball of $\mathbb{C}^{n}$, Springer-Verlag Press, New York, 1980.

[15] K. Zhu, VMO, ESV, and Toeplitz operators on the Bergman space, Trans. Amer. Math. Soc. 302 (1987), 617-646.

[16] ___ Operator Theory in Function Spaces, Marcel Dekker, New York, 1990.

Department of Mathematics

Pusan National University

Pusan 609-735, Korea

E-mail: chohr@pusan.ac.kr

ytseo@naver.com 